\title{
Ultraviolet-Driven Deamination of Cytidine Ribonucleotides Under Planetary Conditions
}

\author{
Zoe R. Todd, ${ }^{1,2}$ Albert C. Fahrenbach, ${ }^{3}$ Sukrit Ranjan, ${ }^{4}$ Christopher J. Magnani, ${ }^{1,2}$ \\ Jack W. Szostak, ${ }^{2}$ and Dimitar D. Sasselov ${ }^{1}$
}

\begin{abstract}
A previously proposed synthesis of pyrimidine ribonucleotides makes use of ultraviolet (UV) light to convert $\beta$-D-ribocytidine-2', $3^{\prime}$-cyclic phosphate to $\beta$-D-ribouridine- $2^{\prime}, 3^{\prime}$-cyclic phosphate, while simultaneously selectively degrading synthetic byproducts. Past studies of the photochemical reactions of pyrimidines have employed mercury arc lamps, characterized by narrowband emission centered at $254 \mathrm{~nm}$, which is not representative of the UV environment of the early Earth. To further assess this process under more realistic circumstances, we investigated the wavelength dependence of the UV-driven conversion of $\beta$-D-ribocytidine$2^{\prime}, 3^{\prime}$-cyclic phosphate to $\beta$-D-ribouridine- $2^{\prime}, 3^{\prime}$-cyclic phosphate. We used constraints provided by planetary environments to assess the implications for pyrimidine nucleotides on the early Earth. We found that the wavelengths of light $(255-285 \mathrm{~nm})$ that most efficiently drive the deamination of $\beta$-D-ribocytidine- $2^{\prime}, 3^{\prime}$-cyclic phosphate to $\beta$-D-ribouridine- $2^{\prime}, 3^{\prime}$-cyclic phosphate are accessible on planetary surfaces such as those of the Hadean-Archaean Earth for $\mathrm{CO}_{2}-\mathrm{N}_{2}$-dominated atmospheres. However, continued irradiation could eventually lead to low levels of ribocytidine in a low-temperature, highly irradiated environment, if production rates are slow. Key Words: Photochemistry-Pyrimidines-Nucleotides_Early Earth. Astrobiology 20, 878-888.
\end{abstract}

\section{Introduction}

T He Elucidation of a potentially prebiotic synthetic pathway for activated pyrimidine ribonucleotides (Powner et al., 2009) from simple starting materials provided one possible solution to a long-term issue with the RNA world: the synthesis of pyrimidine monomers. Recently, substantial progress has been made toward the potentially prebiotic synthesis of canonical and non-canonical RNA nucleosides (Becker et al., 2018) and nucleotides (Powner et al., 2009; Kim and Benner, 2017; Stairs et al., 2017; Xu et al., 2017; Becker et al., 2019).

Powner et al. (2009) achieved the synthesis of $\beta$-Dribocytidine-2', $3^{\prime}$-cyclic phosphate (denoted hereafter as $\mathrm{C}>\mathrm{p}$ ) from simple precursors, that is, glycolaldehyde, D-glyceraldehyde, cyanamide, cyanoacetylene, and phosphate. The irradiation of the mixture that forms $\mathrm{C}>\mathrm{p}$ selectively degrades non-canonical ribonucleotide byproducts and affords partial conversion of $\mathrm{C}>\mathrm{p}$ into $\beta$-D-ribouridine- $2^{\prime}, 3^{\prime}$-cyclic phosphate (denoted as $\mathrm{U}>\mathrm{p}$ ). Later work also utilized partial conversion of
$\mathrm{C}>\mathrm{P}$ to $\mathrm{U}>\mathrm{p}$ under ultraviolet (UV) irradiation, while additionally harnessing advantageous photoanomerization chemistry at an earlier synthetic step to increase the yield of the biological $\beta$-anomer via photoanomerization of $\alpha-2$ thioribocytidine at a 76\% efficiency (Xu et al., 2017).

Powner et al. (2009) and Xu et al. (2017) used mercury arc lamps (primary emission at $254 \mathrm{~nm}$ ) as a source of UV light, but such narrowband emission is not consistent with the spectral flux of the Sun on the surface of the early Earth. The lack of $\mathrm{O}_{2}$ and $\mathrm{O}_{3}$ in the atmosphere would have allowed mid-range UV light $(200-300 \mathrm{~nm})$ to penetrate to the surface of the planet (Cockell, 2000; Ranjan and Sasselov, 2017). This atmospheric scenario begs the question posed by Ranjan and Sasselov (2016): Would the UV photochemistry (here, specifically the partial conversion of $\mathrm{C}>\mathrm{p}$ into $\mathrm{U}>\mathrm{p}$ ) actually occur at a realistic rate under the UV environment on the Hadean Earth? In this study, we address the photochemistry of $\mathrm{C}>$ p over a range of $\mathrm{UV}$ wavelengths and use these data to model the lifetime and concentrations of $\mathrm{C}>\mathrm{p}$ under various environmental conditions.

\footnotetext{
${ }^{1}$ Department of Astronomy, Harvard-Smithsonian Center for Astrophysics, Cambridge, Massachusetts.

${ }^{2}$ Howard Hughes Medical Institute, Department of Molecular Biology and Center for Computational and Integrative Biology, Massachusetts General Hospital, Boston, Massachusetts.

${ }^{3}$ School of Chemistry, University of New South Wales, Sydney, Australia.

${ }^{4}$ SCOL Postdoctoral Fellow, Department of Earth, Atmospheric and Planetary Sciences, Massachusetts Institute of Technology, Cambridge, Massachusetts.

(C) Zoe R. Todd et al., 2020; Published by Mary Ann Liebert, Inc. This Open Access article is distributed under the terms of the Creative Commons License (http://creativecommons.org/licenses/by/4.0), which permits unrestricted use, distribution, and reproduction in any medium, provided the original work is properly credited.
} 
The effect of UV irradiation on the pyrimidine nucleotides/ nucleosides/nucleobases has been the focus of intense study, mostly due to an interest in DNA/RNA damage. In addition to the UV-induced formation of pyrimidine dimers, UV light can cause chemical changes to individual nucleobases, nucleosides, and nucleotides. Early work revealed that UV irradiation of uridine produces a hydrated species, namely 6-hydroxy-5,6-dihydrouridine (Wechter and Smith, 1968; Ducolomb et al., 1976). This photo-generated hydrate can be converted back into uridine thermally or under highly acidic conditions (Sinsheimer and Hastings, 1949). Similarly, the product of UV irradiation of cytidine was postulated to be 6-hydroxy-5,6-dihydrocytidine based on hydrolysis to the uridine hydrate derivative (Johns et al., 1965) and borohydride reduction (Miller and Cerutti, 1968). The irradiation product of cytidine was confirmed to be 6hydroxy-5,6-dihydrocytidine by direct nuclear magnetic resonance (NMR) characterization (Liu and Yang, 1978).

On UV irradiation, the $270 \mathrm{~nm}$ band in the absorption spectrum of cytidine decreases, while a band at $240 \mathrm{~nm}$ appears (Sinsheimer, 1957; Wang, 1959; Wierzchowski and Shugar, 1961), resulting from absorption of the photohydrate, 6-hydroxy-5,6-dihydrocytidine (Fig. 1A). This photohydrate is unstable and can either revert to cytidine or, alternatively, deaminate to generate the uridine photohydrate (Schuster, 1964). The photohydrate of uridine, 6-hydroxy-5,6dihydrouridine, then reverts to uridine, but on longer timescales than the cytidine photohydrate (DeBoer et al., 1970).

Though cytosine, cytidine, and 5'-CMP form a photohydrated intermediate on UV irradiation, $\mathrm{C}>\mathrm{p}$ was hypothesized by Powner et al. (2009) to form a different intermediate, in which the $5^{\prime}$-hydroxyl adds intramolecularly to C6 to give a bridged ether (Fig. 1B). Powner et al. (2009) hypothesized that elimination of the $5^{\prime}-\mathrm{OH}$ in the postulated photoproduct is a slower process in comparison to the dehydration of the analogous photohydrate, causing the $\mathrm{C}>\mathrm{p}$ to undergo fewer cycles of photoexcitation, allowing some degree of protection from UV damage. Powner et al. (2009) observed that $\mathrm{C}>\mathrm{p}$ is more stable to UV photodegradation than other cytosine nucleotides and nucleosides, including $\alpha$-ribocytidine- $5^{\prime}$-phosphate, $\beta$-arabinocytidine, $\beta$ arabinocytidine- $5^{\prime}$-phosphate, and $\alpha$-ribocytidine- $2^{\prime}, 3^{\prime}$-cyclic phosphate, suggesting that UV may have played a role in selecting the canonical nucleotide stereochemistry due to increased stability. This is not the only suggestion of UV light playing a role in selection; in addition, the canonical nucleobases have shorter excited state lifetimes on UV irradiation than some other non-canonical bases (Beckstead et al., 2016). Again, this promotes increased stability toward photoreactions of the canonical nucleobases, suggesting that UV potentially acted as a selection pressure on the early Earth (Lazcano et al., 1988; Rios and Tor, 2013; Xu et al., 2017).

In this study, we do not attempt to differentiate between the two possible intermediates, the photohydrate or the bridged 5'-6 cyclic molecule (Fig. 1), as the identity of the intermediate is not critical to the overall implications for the final products of the reaction and will not change our results. We focus on the UV-mediated partial conversion of $\beta$-D-ribocytidine- $2^{\prime}, 3^{\prime}$-cyclic phosphate to $\beta$-D-ribouridine- $2^{\prime}$, $3^{\prime}$-cyclic phosphate. In particular, we examine the UV wavelength dependence of this reaction in the context of the UV environment on the early Earth to assess the planetary

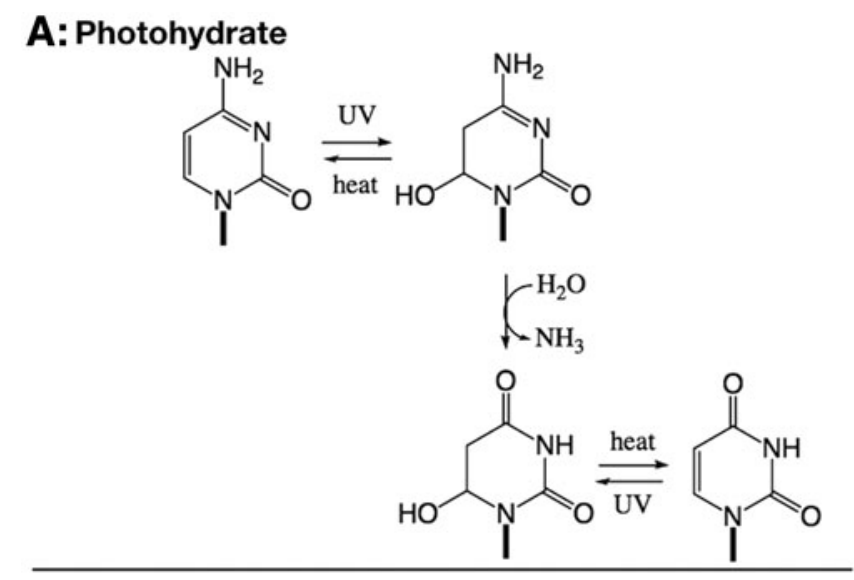

B:6,5'-ether linked intermediate

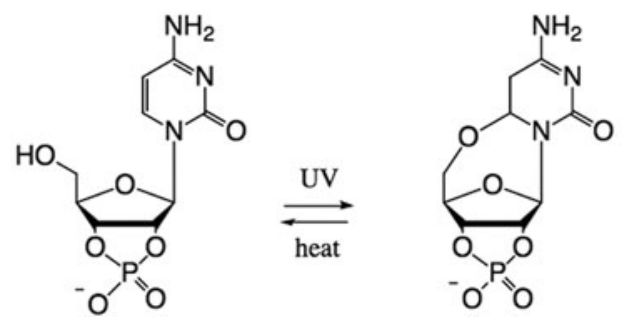

FIG. 1. Two potential mechanisms for the photochemical processing of $\beta$-D-ribocytidine-2', $3^{\prime}$-cyclic phosphate. (A) Mechanism for cytidine, cytosine, and 5'-CMP, where UV irradiation produces the photohydrate (e.g., 6-hydroxy5,6-dihydrocytosine). This photohydrate can undergo deamination to the corresponding uridine photohydrate. Both photohydrates can be converted into the canonical nucleobase through thermal dehydration (the rate of thermal recovery of C is much greater than that of U). (B) Structure of the hypothesized intermediate on UV irradiation of $\beta$-Dribocytidine- $2^{\prime}, 3^{\prime}$-cyclic phosphate, suggested by Powner et al. (2009). Similarly, this intermediate could undergo deamination and then thermal recovery to $\beta$-D-ribouridine$2^{\prime}, 3^{\prime}$-cyclic phosphate. UV, ultraviolet.

implications for this reaction. We then use these data to model the concentrations and lifetimes of $\mathrm{C}>\mathrm{p}$ for different environments on the early Earth.

\section{Materials and Methods}

To investigate the UV-driven conversion of $\mathrm{C}>\mathrm{p}$, a $50 \mu \mathrm{M}$ solution of $\mathrm{C}>\mathrm{p}$ in degassed, deionized water was prepared. Aliquots of this solution were irradiated from 215 to $295 \mathrm{~nm}$ individually in $10-\mathrm{nm}$ internals with a $10-\mathrm{nm}$ bandwidth. To perform the irradiation, a xenon arc lamp coupled with a diffraction grating acting as a monochromator to allow for tunable wavelength selection was used (as in Todd et al., 2018). The flux from the lamp over the wavelength interval $265-285 \mathrm{~nm}$ is within an order of magnitude of the surface flux expected over the same wavelength range on the surface of the early Earth (Ranjan and Sasselov, 2017).

Solutions were monitored by UV-Vis spectroscopy at 15min intervals throughout irradiation, for a total duration of $2 \mathrm{~h}$. On irradiation, the absorption band centered at $270 \mathrm{~nm}$ from $C>p$ decreases and an absorption band at $240 \mathrm{~nm}$ grows in. These spectral changes were used to determine the 
concentrations of the starting material and photogenerated intermediate as a function of irradiation time, through experimentally determined extinction coefficients for both species (with concentrations determined by ${ }^{31} \mathrm{P}-\mathrm{NMR}$, see Appendix A1, Figure A1). Once extinction coefficients were determined, the concentrations of the two species were determined from the UV absorption at the maximum wavelengths for the intermediate and starting material by solving a system of two equations, as described below:

$$
\begin{aligned}
& A_{240 \mathrm{~nm}}=\epsilon_{\mathrm{C}>\mathrm{p}, 240 \mathrm{~nm}} c_{\mathrm{C}>\mathrm{p}} l+\epsilon_{\mathrm{int}, 240 \mathrm{~nm}} c_{\text {int }} l \\
& A_{270 \mathrm{~nm}}=\epsilon_{\mathrm{C}>\mathrm{p}, 270 \mathrm{~nm}} c_{\mathrm{C}>\mathrm{p}} l+\epsilon_{\mathrm{int}, 270 \mathrm{~nm}} c_{\text {int }} l
\end{aligned}
$$

We then determined the observed rate constant of the reaction by plotting the logarithm of concentration against time for each wavelength. Irradiations at each wavelength were performed in triplicate, to obtain an average rate constant and error. To compare reaction rates at different wavelengths, the observed rate constants were normalized by photon flux to get the rate constant, since the lamp used does not provide constant fluxes at all wavelengths. To do this normalization, powers at each wavelength were measured with a Newport power detector; they were then converted to a photon flux through the relation between wavelength and energy of a photon.

For experimental testing, the thermal reversion of the photoproduct of $\mathrm{C}>\mathrm{p}$, solutions of $50 \mu \mathrm{M} \mathrm{C}>\mathrm{p}$ were irradiated in a Rayonet reactor (RPR-200, $254 \mathrm{~nm}$, mercury lamps) for $15 \mathrm{~min}$. Cuvettes were then placed in a Cary UVVis spectrometer with adjustable temperature control. Temperatures were varied from $25^{\circ} \mathrm{C}$ to $45^{\circ} \mathrm{C}$, in $5^{\circ}$ intervals. Each temperature tested was monitored for $>16 \mathrm{~h}$. UVVis absorption spectra were recorded and used to calculate concentrations to then determine reversion rates.

To study the partitioning of the photochemically generated intermediate between $\mathrm{C}>\mathrm{p}$ and $\mathrm{U}>\mathrm{p}$, solutions of $50 \mu \mathrm{M} \beta$-ribocytidine- $2^{\prime}, 3^{\prime}$-cyclic phosphate were irradiated for varying irradiation times $(0,10,30,60,90$, and $120 \mathrm{~min})$ in the Rayonet reactor at $254 \mathrm{~nm}$. After the set irradiation time elapsed, the samples were placed in a Cary UV-Vis spectrometer with an adjustable temperature control. Cuvettes were heated at $60^{\circ} \mathrm{C}$ for $100 \mathrm{~h}$ total, with UV-Vis monitoring every $30 \mathrm{~min}$. The concentrations of $\mathrm{C}>\mathrm{p}, \mathrm{U}>\mathrm{p}$, and the photochemically generated intermediate were determined from solving a system of three equations from the absorption values at the maximum absorption wavelengths of the three species $(240,260$, and $270 \mathrm{~nm}$ for the intermediate, $\mathrm{U}>\mathrm{p}$, and $\mathrm{C}>\mathrm{p}$, respectively):

$$
\begin{aligned}
& A_{240 \mathrm{~nm}}=\epsilon_{\mathrm{C}>\mathrm{p}, 240 \mathrm{~nm}} c_{\mathrm{C}>\mathrm{p}} l+\epsilon_{\mathrm{int}, 240 \mathrm{~nm}} c_{\mathrm{int}} l+\epsilon_{\mathrm{U}>\mathrm{p}, 240 \mathrm{~nm}} c_{\mathrm{U}>\mathrm{p}} l \\
& A_{260 \mathrm{~nm}}=\epsilon_{\mathrm{C}>\mathrm{p}, 260 \mathrm{~nm}} c_{\mathrm{C}>\mathrm{p}} 1+\epsilon_{\mathrm{int}, 260 \mathrm{~nm}} c_{\mathrm{int}} l+\epsilon_{\mathrm{U}>\mathrm{p}, 260 \mathrm{~nm}} c_{\mathrm{U}>\mathrm{p}} l \\
& A_{270 \mathrm{~nm}}=\epsilon_{\mathrm{C}>\mathrm{p}, 270 \mathrm{~nm}} c_{\mathrm{C}>\mathrm{p}} l+\epsilon_{\mathrm{int}, 270 \mathrm{~nm}} c_{\mathrm{int}} l+\epsilon_{\mathrm{U}>\mathrm{p}, 270 \mathrm{~nm}} c_{\mathrm{U}>\mathrm{p}} l
\end{aligned}
$$

We note that the reaction likely contains another species: the uridine form of the intermediate. $\mathrm{C}>\mathrm{p}$ photochemically generates the cytidine intermediate (either cytidine photohydrate or cytidine bridged ether intermediate, see Fig. 1). This structure then deaminates to give the uridine form of the intermediate. Thermal recovery generates $U>p$ from any deaminated intermediate while regenerating $\mathrm{C}>\mathrm{p}$ from any cytidine intermediate that did not deaminate. The uridine form of the intermediate does not absorb significantly in the 230-300 $\mathrm{nm}$ range (Appendix A2), so it should not interfere with the concentration determinations through UV absorption measurements.

\section{Results}

\subsection{Wavelength-dependent UV conversion}

Irradiation of $50 \mu \mathrm{M} \mathrm{C}>\mathrm{p}$ causes the initial absorption feature at $270 \mathrm{~nm}$ to decrease, whereas a band at $240 \mathrm{~nm}$ grows in (Fig. 2A). A clear isosbestic point is observed at $\sim 250 \mathrm{~nm}$. This observation suggests that $\mathrm{C}>\mathrm{p}$ is directly converted to the photoproduct, and only one photoproduct is formed initially. ${ }^{31} \mathrm{P}-\mathrm{NMR}$ spectroscopy supports this supposition by the fact that irradiation of $C>p$ initially results only in one detectable ${ }^{31} \mathrm{P}$ resonance other than the starting material. These spectral changes, coupled with experimentally determined extinction coefficients for the starting material and photoproduct (Appendix A1), can be used to determine their concentrations during irradiation. The slope of the best fit line for $\ln ([\mathrm{C}>\mathrm{p}])$ as a function of time gives the observed rate constant (Fig. 2B). Solutions were irradiated at $215-295 \mathrm{~nm}$ (10-nm interval, $10-\mathrm{nm}$ bandwidth) in triplicate. Figure 3 shows the rate constant
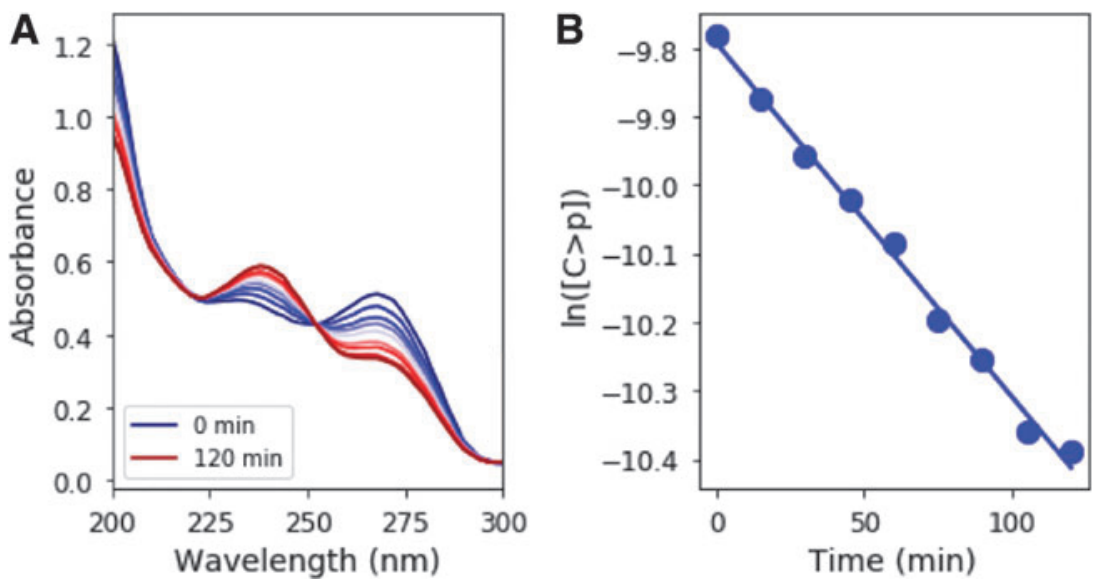

FIG. 2. (A) UV absorption spectrum with irradiation at $265 \mathrm{~nm}$ in the tunable lamp setup. As irradiation is continued, the absorption at $240 \mathrm{~nm}$ increases whereas that at $270 \mathrm{~nm}$ decreases. This observation is due to the accumulation of intermediate and depletion of starting material, respectively. The concentrations of starting material and intermediate can be calculated for each time point from the UV-Vis absorption spectra. (B) Plot of logarithm of concentration of $\mathrm{C}>\mathrm{p}$ with irradiation time. The slope of the best-fit line gives the observed rate constant. 


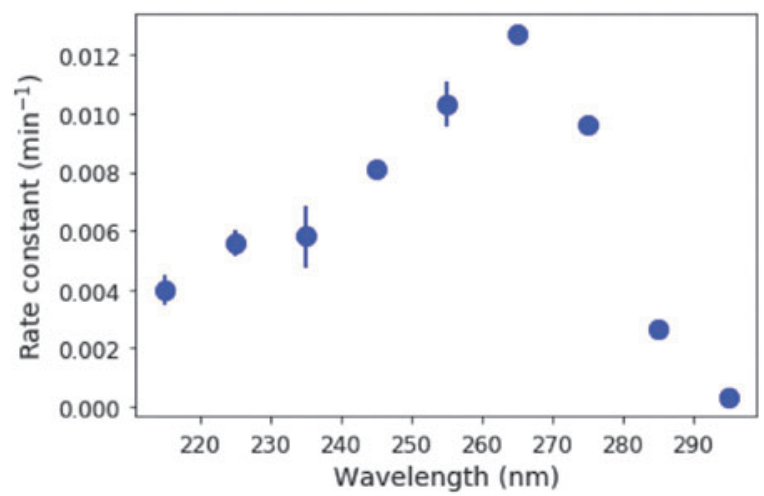

FIG. 3. Rate constant of UV-driven reaction of $\mathrm{C}>\mathrm{p}$ to the intermediate photoproduct as a function of irradiation wavelength, for a constant photon flux from 210 to $300 \mathrm{~nm}$. The rate constant is greatest at a wavelength of $265 \mathrm{~nm}$, as might be expected from the maximum absorption of $\mathrm{C}>\mathrm{p}$ near this wavelength.

[for a constant photon flux of $5 \times 10^{14} \mathrm{phot} / \mathrm{s} / \mathrm{cm}^{2}$, expected from 210 to $300 \mathrm{~nm}$ based on the baseline early Earth scenario from (Ranjan and Sasselov, 2017), which uses atmospheric profiles from Rugheimer et al. (2015)] across a range of irradiation wavelengths, with the points representing the average of the triplicate set and the errors estimated by the standard deviation. The rate constant is maximum at $265 \mathrm{~nm}$, which is consistent with expectations based on the absorption maximum of the starting material. The rate constant decreases significantly at wavelengths shorter than $245 \mathrm{~nm}$ and longer than $275 \mathrm{~nm}$.

We next sought to compare the experimentally determined rate constants at various irradiation wavelengths with the spectral flux available on the surface of the early Earth from the Sun. A two-stream radiative transfer model produced and described in the work of Ranjan and Sasselov (2017) was used to calculate the surface spectral radiance through a sample $\mathrm{N}_{2} / \mathrm{CO}_{2}$-dominated $\left(0.9\right.$ bar $\mathrm{N}_{2}, 0.1$ bar $\mathrm{CO}_{2}$ ) prebiotic atmosphere; the surface UV environment is robust to the uncertainties in the early Earth's atmospheric state as a result of saturation of absorption of wavelengths $<204 \mathrm{~nm}$ due to $\mathrm{CO}_{2}$, resulting in mid-range UV wavelengths from $\sim 200$ to $300 \mathrm{~nm}$ present on the surface of the planet (Ranjan and Sasselov, 2016, 2017). The intensities of longer wavelength UV light are greater than those of shorter wavelengths on the surface of the planet (see green line in Fig. 4). We then integrated the surface radiance by using the same $10-\mathrm{nm}$ bins that the experiments employed, and calculated the relative rate of the reaction, defined as the product of the experimental rate and the integrated surface radiance (blue points in Fig. 4). The relative rate is still maximum at $265 \mathrm{~nm}$, and the most productive wavelengths for driving this photochemical reaction in our model for the surface of the early Earth are 255-285 nm.

\subsection{Thermal reversion}

On continued UV irradiation of $\mathrm{C}>\mathrm{p}$, the photochemically generated intermediate can either revert to $\mathrm{C}>\mathrm{p}$ thermally, deaminate to form the uridine-derivative of the photochemically generated intermediate, or possibly undergo a second photochemical reaction. The photochemically generated uridine intermediate is more thermally stable than the cytidine photochemically generated intermediate (DeBoer et al., 1970), and hence the production of $\mathrm{U}>\mathrm{p}$ requires prolonged heating. We investigated the thermal reversion of the UV-generated intermediate back to $\mathrm{C}>\mathrm{p}$ at different temperatures to determine the activation energy for this reaction: $50 \mu \mathrm{M} \mathrm{C}>\mathrm{p}$ solutions were irradiated in a RPR-200 Rayonet reactor $(254 \mathrm{~nm})$ for $15 \mathrm{~min}$, until they were converted to at least $90 \%$ intermediate (as determined by UVVis spectroscopy). Then, solutions were incubated in the dark at temperatures from $25^{\circ} \mathrm{C}$ to $45^{\circ} \mathrm{C}$, in $5^{\circ}$ intervals for $16-24 \mathrm{~h}$, while being monitored every $15-30 \mathrm{~min}$ by UV-Vis absorption spectroscopy. Concentrations of the starting material and UV-generated intermediate were determined and fit with exponentials as a function of incubation time. Figure 5A shows the concentration of the intermediate and starting material during heating at $35^{\circ} \mathrm{C}$. The rates for thermal reversion to $\mathrm{C}>\mathrm{p}$ were determined for various temperatures to generate an Arrhenius plot (Fig. 5B). The activation energy for this reaction was determined from both the appearance of $\mathrm{C}>\mathrm{p}$ and the disappearance of the intermediate, as $84.0 \pm 10.5$ and $86.2 \pm 11.7 \mathrm{~kJ} / \mathrm{mol}$, respectively, that is, the same within error. These were determined in the same reaction, with rates and activation energies calculated from the increase in $\mathrm{C}>\mathrm{p}$ and the decrease in the intermediate, respectively. We did not see the appearance of $U>p$ during the course of these experiments, since this requires elevated temperatures for longer periods of time.

\subsection{Cytidine-derivative versus uridine-derivative partitioning}

On UV irradiation of $\mathrm{C}>\mathrm{p}$, the photochemically generated intermediate can deaminate to give the uridine form of the intermediate, which is more thermally stable to dehydration, and subsequent recovery to the canonical nucleotide than the cytidine intermediate. Accordingly, we next attempted to see the generation of $\mathrm{U}>\mathrm{p}$ by irradiating $\mathrm{C}>\mathrm{p}$ and then incubating the solution for a prolonged period at

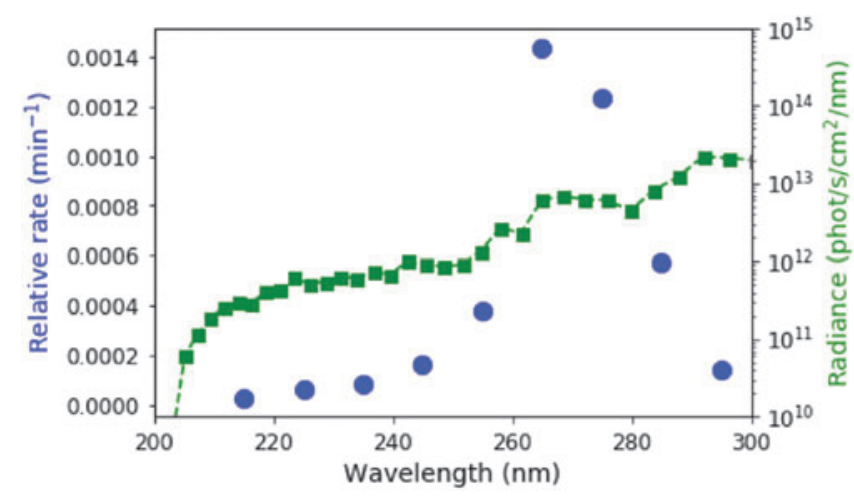

FIG. 4. The relative rate of the conversion of $\mathrm{C}>\mathrm{p}$ into the photochemically generated intermediate, taking into account the spectral surface flux (green squares) and the relative rate constants at varying wavelengths are shown in the blue points. The relative rate is maximum at $265 \mathrm{~nm}$. The effective range of radiation for driving this reaction is from roughly $255-285 \mathrm{~nm}$. 

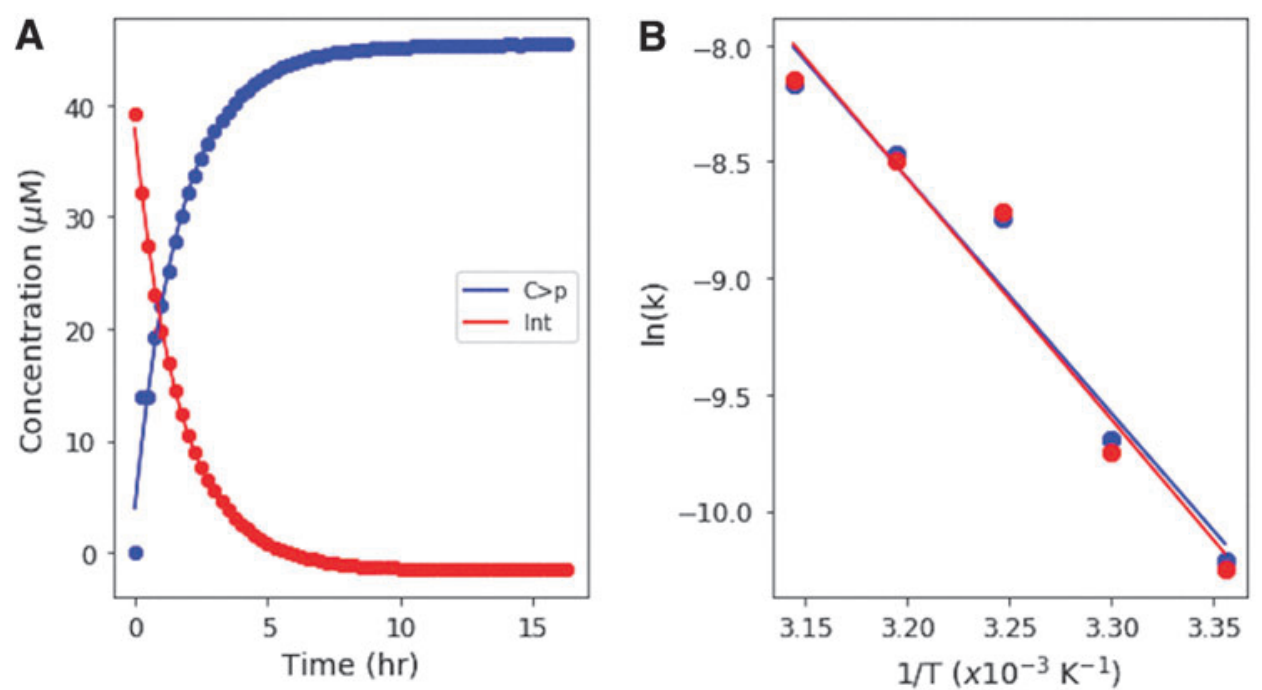

FIG. 5. (A) Concentrations of $\mathrm{C}>\mathrm{p}$ and the photogenerated intermediate during thermal recovery at $35^{\circ} \mathrm{C}$. The concentrations are fit by exponential functions, which allow the rate constant of recovery to be calculated for a given temperature. (B) Arrhenius plot for both the rate of recovery of $\mathrm{C}$ and the rate of disappearance of the intermediate. The logarithm of the rate constant is plotted as a function of inverse temperature. The activation energies can be calculated from the slopes of the trendlines, giving activation energies of $84.0 \pm 10.5$ and $86.2 \pm 11.7 \mathrm{~kJ} / \mathrm{mol}$ for $\mathrm{C}>\mathrm{p}$ and the intermediate, respectively, that is, the same within error.

elevated temperatures. A solution of $50 \mu \mathrm{M} \mathrm{C}>\mathrm{p}$ was irradiated for $90 \mathrm{~min}$ in the Rayonet RPR-200 reactor $(254 \mathrm{~nm})$; then, it was allowed to sit in the dark at $60^{\circ} \mathrm{C}$ for $100 \mathrm{~h}$. Figure 6A shows the UV-Vis absorption spectra of this solution during the heating period after irradiation. The concentrations of $\mathrm{C}>\mathrm{p}$, the photochemically generated cytidine intermediate, and $U>p$ were extracted from the UV-Vis spectra throughout the incubation and are shown in Fig. 6B. The intermediate is quickly returned to $\mathrm{C}>\mathrm{p}$, whereas it takes longer for $U>p$ to appear, likely due to the uridine photochemically generated intermediate is more thermally stable. We do not attempt to quantify the uridine intermediate, as it does not show a clear absorption peak. It does not absorb significantly in the 230-300 nm window (Appendix A2), so the presence of this species in the reaction should not affect our overall determinations of the concentrations of the other species that are absorbed through the UV spectra.
After $100 \mathrm{~h}$ at $60^{\circ} \mathrm{C}$, the fractions of $\mathrm{C}>\mathrm{p}$ and $\mathrm{U}>\mathrm{p}$ reach a constant ratio. To better understand the partitioning between $\mathrm{C}>\mathrm{p}$ and $\mathrm{U}>\mathrm{p}$ and the role of irradiation, we allowed the initial irradiation time before incubation to vary. After irradiation (for $0,10,30,60,90$, or $120 \mathrm{~min}$ ), samples were incubated at $60^{\circ} \mathrm{C}$ for $100 \mathrm{~h}$ and monitored by UV-Vis. Figure 7 shows the final concentration of $C>p$ and $U>p$ after heating $\left(100 \mathrm{~h}, 60^{\circ} \mathrm{C}\right)$ at various irradiation times. $\mathrm{C}>\mathrm{p}$ is depleted more with increasing irradiation times, whereas the level of $U>$ p after incubation seems to be approximately constant across different irradiation times. At the longer irradiation times, the proportion of $\mathrm{C}>\mathrm{p}$ and $\mathrm{U}>\mathrm{p}$ reaches a value of roughly $61 \% \mathrm{C}$ and $33 \% \mathrm{U}$, though the total amount of material decreases with increasing irradiation times. This indicates that prolonged irradiation will deplete $\mathrm{C}>\mathrm{p}$, and it may also limit the amount of $U>p$ generated, if production rates are slow.
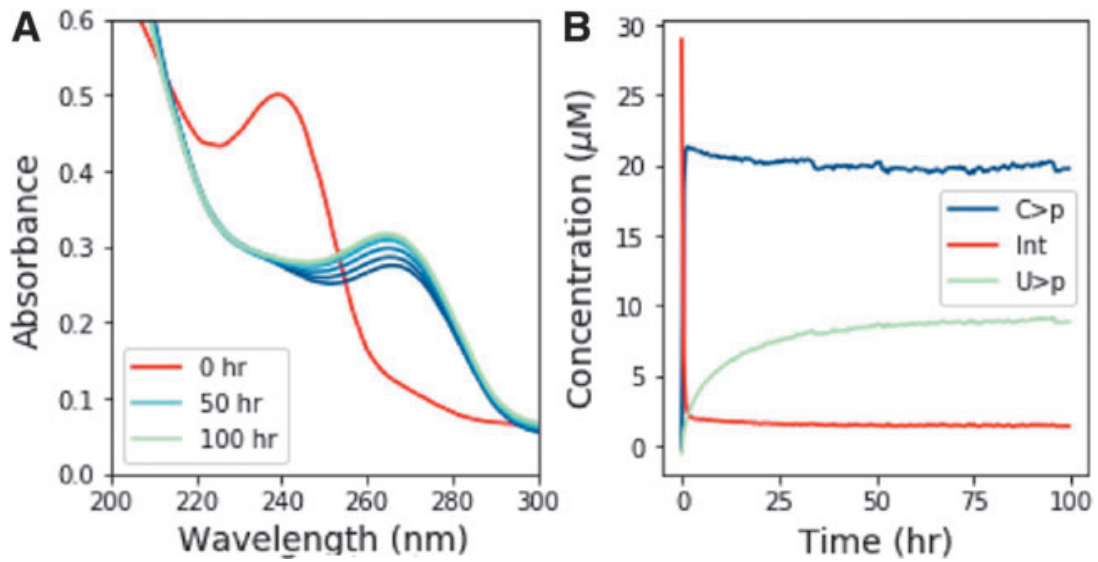

FIG. 6. (A) Absorption spectra for various incubation times at $60^{\circ} \mathrm{C}$ after irradiation at $254 \mathrm{~nm}$ for $90 \mathrm{~min}$. (B) Concentration of intermediate, $\mathrm{C}>\mathrm{p}$, and $\mathrm{U}>\mathrm{p}$ as a function of incubation time, extracted from the absorption spectra. As heating is continued, the absorption spectra show increasing absorption at $260 \mathrm{~nm}$, consistent with a thermal recovery of the deaminated intermediate to $\beta$ D-ribouridine- $2^{\prime}, 3^{\prime}$-cyclic phosphate. 


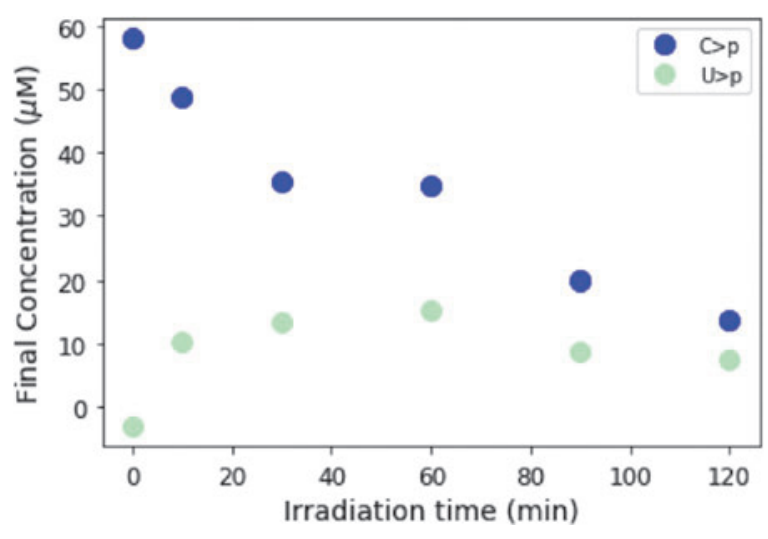

FIG. 7. Final concentration of $\mathrm{C}>\mathrm{p}$ and $\mathrm{U}>\mathrm{p}$ after $100 \mathrm{~h}$ of incubation at $60^{\circ} \mathrm{C}$, after irradiation for varying amounts of time $(0,10,30,60,90,120 \mathrm{~min})$. During this longer heating step, the absorption spectra show increasing absorption at $260 \mathrm{~nm}$, which indicates a recovery of the uridine intermediate to $U>p$. Longer irradiation times lead to an overall loss of material that is likely due to irreversible photodamage. At longer irradiation times, the ratios of $\mathrm{C}>\mathrm{p}$ to $\mathrm{U}>\mathrm{p}$ appear to plateau near $61 \% \mathrm{C}>\mathrm{p}$ and $33 \% \mathrm{U}>\mathrm{p}$, after $100 \mathrm{~h}$ of incubation at $60^{\circ} \mathrm{C}$.

\subsection{Model day/night cycle}

We next studied what happens to $\mathrm{C}>\mathrm{p}$ under repeated photocycling/thermocycling. We began with $50 \mu \mathrm{M} \mathrm{C}>\mathrm{p}$ and alternated between irradiating in the Rayonet reactor $(254 \mathrm{~nm})$ for $15 \mathrm{~min}$ and incubating at $35^{\circ} \mathrm{C}$ for $24 \mathrm{~h}$, repeating for a total of eight cycles. Figure 8A shows the absorption spectra for the solution immediately after each irradiation cycle, with the absorption spectrum of the starting material (before irradiation) shown by the dashed black line. Figure 8B shows the absorption spectra after the completion of the thermal step $\left(24 \mathrm{~h}\right.$ at $35^{\circ} \mathrm{C}$ after irradiation). With repeated cycles of irradiation and thermal re- covery, material is lost, as seen by the decreasing absorption intensities. These spectra were converted to concentrations of $\mathrm{C}>\mathrm{p}$ and the photochemically generated intermediate after each cycle (Fig. 8C). On average, only $88 \%$ of the material is recovered after a given cycle, leading to significant depletion of $\mathrm{C}>\mathrm{p}$ by the end of the eighth cycle. The lost material is likely converted to the corresponding uridine intermediate or other irreversible photodamage. $U>p$ is not observed under these experimental conditions, since generation of $U>p$ from the uridine intermediate requires elevated temperatures for prolonged periods.

This loss of material leads to a potential limitation of UV deamination of pyrimidine ribonucleotides. If a fixed stock of $\mathrm{C}>\mathrm{p}$ undergoes repeated cycles of irradiation, it will ultimately be lost to $U>p$ or other photoproducts, limiting the overall amount of $\mathrm{C}>\mathrm{p}$ that could be available for prebiotic chemistry. This situation would be mitigated if the production of $\mathrm{C}>\mathrm{p}$ occurs at a similar rate to the UVinduced loss. We asked what production rates of $\mathrm{C}>\mathrm{p}$ are needed to sustain a steady state under a variety of planetary environmental conditions by simulating a day-night cycle expected on the early Earth (16h total, e.g., Lathe, 2006). Figure 9 shows the concentration of $\mathrm{C}>\mathrm{p}$ (initially assumed to be $1 \mathrm{mM}$ ) under simulated day-night cycles. The net irradiation loss rate is calculated by weighting the experimentally determined rate constants by the solar irradiation flux calculated for the surface of the early Earth, and then integrating over $210-300 \mathrm{~nm}$. The photochemical rate is assumed to be temperature independent, whereas the dark reaction is not. The thermal recovery during the dark is modeled at three temperatures: $15^{\circ} \mathrm{C}, 25^{\circ} \mathrm{C}$, and $35^{\circ} \mathrm{C}$. With each cycle, we impose a maximum recovery of $88 \%$ $\beta$-ribocytidine- $2^{\prime}, 3^{\prime}$-cyclic phosphate, as indicated by our experimental results. This repeated photocycling depletes the initial stock of $\mathrm{C}>\mathrm{p}$ on varying timescales depending on the temperature, where higher temperatures are more favorable for longer residence times of $\mathrm{C}>\mathrm{p}$. The yellow shaded region in Fig. 9 indicates levels of $\mathrm{C}>p>1 \mu \mathrm{M}$,
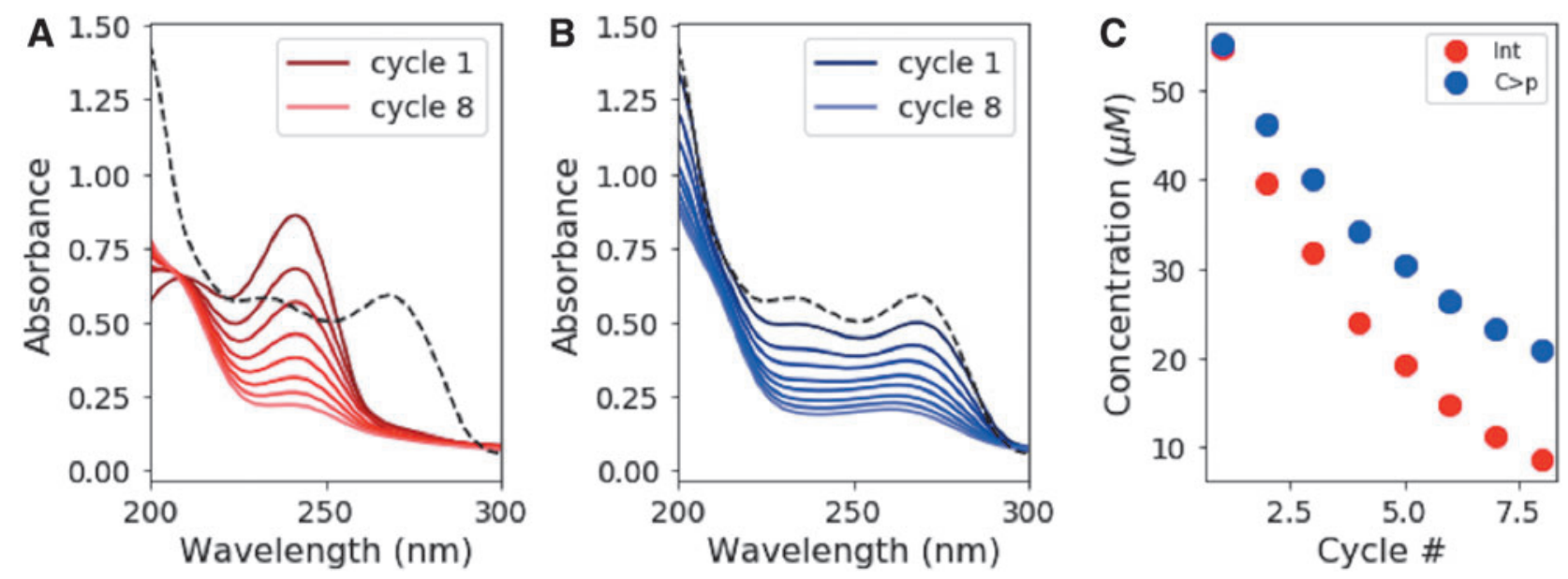

FIG. 8. Degradation of material over repeated cycles of irradiation and thermal recovery. (A) Absorption spectra of the irradiated species (primarily intermediate) after each 15-min irradiation cycle. Each irradiation is followed by thermal recovery to make one full cycle. (B) Absorption spectra of the recovered (primarily starting) material as a function of cycle, after $24 \mathrm{~h}$ at $35^{\circ} \mathrm{C}$. The black dotted line shows the absorption spectrum of the initial material (before irradiation). (C) Concentration of the intermediate and $\mathrm{C}>\mathrm{p}$ after irradiation and thermal recovery, respectively, as a function of cycle number. With repeated cycles, material is lost; leaving roughly $88 \%$ of $\mathrm{C}>\mathrm{p}$ recovered with each successive cycle. 


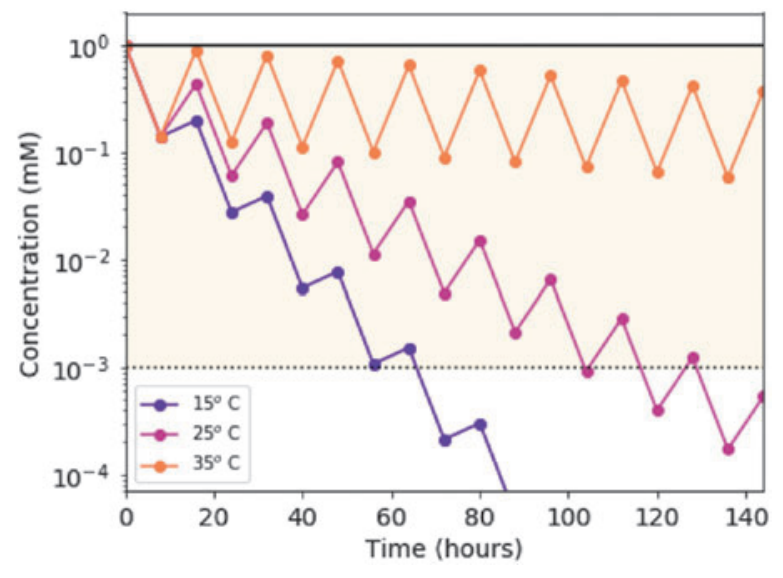

FIG. 9. Model of the concentration of $\mathrm{C}>\mathrm{p}$ through successive day/night cycles $(16 \mathrm{~h})$ from the experimentally determined wavelength- and temperature-dependent rates. The concentration of $\mathrm{C}>\mathrm{p}$ is calculated as a function of time for temperatures of $15^{\circ} \mathrm{C}, 25^{\circ} \mathrm{C}$, and $35^{\circ} \mathrm{C}$. The irradiation degradation rate is determined by weighting the measured rate constants by the solar irradiation flux expected on the surface of the early Earth and integrating from 210 to $300 \mathrm{~nm}$. We include degradation, by only allowing $88 \%$ of $\mathrm{C}>\mathrm{p}$ to return with each successive cycle, leading to an overall loss with time. During these successive irradiation cycles, we would expect accumulation of the corresponding uridine intermediate, which could then be recovered as $U>p$ on prolonged heating. The yellow shaded region indicates concentrations above $1 \mu \mathrm{M}$, which may be enough for prebiotic chemistry to occur.

which is the threshold where prebiotic chemistry is believed to be plausible. $\mathrm{C}>\mathrm{p}$ (initially at $1 \mathrm{mM}$ ) is expected to be $>1 \mu \mathrm{M}$ for 68,130 , and $1010 \mathrm{~h}$ for $T=15^{\circ} \mathrm{C}, 25^{\circ} \mathrm{C}$, and $35^{\circ} \mathrm{C}$, respectively. We find these results to be insensitive to the assumed length of the day.

This model can also be used to determine the production rates that are necessary to sustain a steady state of $\mathrm{C}>\mathrm{p}$ at various concentrations, by equating the production and destruction rates. To maintain $0.1 \mathrm{mM} \mathrm{C}>\mathrm{p}$, production rates need to be from 0.3 to $4.4 \mu \mathrm{M} / \mathrm{h}$, depending on temperature (Table 1). The required production rate scales linearly with steady-state concentration, for example, to obtain $1 \mathrm{mM} \mathrm{C}>\mathrm{p}$ at steady state, production rates need to be $3.0-44 \mu \mathrm{M} / \mathrm{h}$ for the temperature range of $15-35^{\circ} \mathrm{C}$. Past work on the prebiotic synthesis of molecules, including these nucleotides, has generally not focused on rates, but rather yields. These production rates offer a rough guideline as to what is required for the prebiotic chemistry to maintain a fairly constant stock of material without significant depletion under self-consistent conditions, and without invoking additional environmental constraints.

\section{Discussion}

Given that the spectral surface radiance on the early Earth is quite broad, and thus not well represented by narrowband irradiation sources typically used in irradiation experiments, it is important to analyze potential prebiotic photochemical reactions for their plausibility in the context of the UV environment on the early Earth. Previous studies showed a
Table 1. Production Rate of $\mathrm{C}>\mathrm{P}$ Necessary to Maintain a Steady-State Concentration OF 0.1 MM, FOR THREE DIFFERENT TEMPERATURES

\begin{tabular}{|c|c|}
\hline Temperature $\left({ }^{\circ} \mathrm{C}\right)$ & $\begin{array}{c}{[C>p]=0.1 \mathrm{mM} \text { steady state }} \\
\text { Production rate }(\mu M / \mathrm{h})\end{array}$ \\
\hline 15 & 4.4 \\
\hline 25 & 2.3 \\
\hline 35 & 0.3 \\
\hline
\end{tabular}

Lower temperature environments require larger production rates. Production rates scale linearly with steady-state concentration, for example, a steady-state concentration of $1 \mathrm{mM} \mathrm{C}>\mathrm{p}$ requires production rates of $23 \mu \mathrm{M} / \mathrm{h}$ at $25^{\circ} \mathrm{C}$.

prebiotically plausible synthesis of $\beta$-D-ribocytidine- $2^{\prime}, 3^{\prime}$ cyclic phosphate and subsequent UV-driven conversion to $\beta$ D-ribouridine- $2^{\prime}, 3^{\prime}$-cyclic phosphate. The UV light $(254 \mathrm{~nm}$ narrowband emission) also acted to destroy other synthetic co-products, enriching the relative concentration of the canonical ribonucleotides used by life today. To assess the prebiotic plausibility of the conversion of the $C>p$ to $U>p$, we first studied the wavelength dependence of the reaction rate, as monitored by UV-Vis absorption spectroscopy. This analysis showed that the photochemical step proceeded best at an irradiation wavelength of $265 \mathrm{~nm}$. In the context of the spectral flux available on the surface of the early Earth, generally, longer wavelengths are more accessible, meaning the reaction is more efficient under more realistic conditions. To quantify the effect of varying amounts of surface radiation at different wavelengths, we computed the weighted surface intensity, which accounts for the rate of the reaction as a function of wavelength and the intensity of the radiation available on the surface of the early Earth at each wavelength. Two hundred sixty-five nanometers are still the most efficient wavelength, with the window of usable radiation for this reaction occurring around $255-285 \mathrm{~nm}$. Outside this window, either the reaction rate or the radiation intensity drops low enough that the weighted surface intensity suffers significantly. It is fortuitous that the most efficient wavelengths for this reaction are those that are not significantly screened out by plausible prebiotic atmospheric constituents and are available at sufficient intensity from the early Sun to drive the reaction. Our study demonstrates that the wavelengths that are necessary to drive this reaction would likely be available on the surface of the early Earth.

We further investigated the second step of the reaction, which is the thermal reversion of the photochemically generated intermediate back into the starting material. We determined the rate of the back reaction as a function of temperature and found the activation energy to be $85 \pm 10 \mathrm{~kJ} / \mathrm{mol}$. On the prebiotic Earth, materials would be subjected to repeated cycles of light and dark during the day/night cycles. We modeled this by determining the net photochemical rate weighted over the wavelength intensities expected on the early Earth. After the irradiation step, we then modeled the dark reaction at various temperatures. Included in this model was the experimentally determined recovery of, on average, $88 \%$ of the starting material with each cycle. This ultimately leads to a limit on how long $\mathrm{C}>\mathrm{p}$ would have been available on the early Earth, if $\mathrm{C}>\mathrm{p}$ was only present in a finite amount. This calculation can 
conversely be used to constrain the necessary production rate of $\mathrm{C}>\mathrm{p}$ to maintain steady-state concentrations. We find that a stock of $1 \mathrm{mM} \mathrm{C}>\mathrm{p}$ will last from $\sim 70$ to $1000 \mathrm{~h}$ at temperatures of $15-35^{\circ} \mathrm{C}$, and that production rates of $3-44 \mu \mathrm{M} / \mathrm{h}$ are needed to maintain a $1 \mathrm{mM}$ stock of $\mathrm{C}>\mathrm{p}$ (depending on temperature). These considerations can help constrain the environmental conditions for the prebiotic chemistry to be self-consistent and plausible. In particular, if future experiments can quantify typical production rates under plausible planetary conditions, these can be compared to our findings to assess the overall consistency of the proposed chemistry. We also note that the deaminated product that forms as a result of UV light requires a significant amount of heating to return to $\mathrm{U}>\mathrm{p}$. This step would need to occur in the dark on the early Earth to not drive the reaction back to the UV-generated intermediate state. This condition again may imply constraints and requirements on the prebiotic environment if both $\mathrm{C}>\mathrm{p}$ and $\mathrm{U}>\mathrm{p}$ nucleotides are to be obtained through this UV-driven method. In particular, if both $\mathrm{C}>\mathrm{p}$ and $\mathrm{U}>\mathrm{p}$ are to be obtained through $\mathrm{UV}$-driven deamination, the products would need to be incubated in the dark or exposed to significant durations of heating to recover the canonical ribonucleotides.

With these experimental constraints on this prebiotic chemical reaction, we can begin to envision an environmental scenario in which photochemistry can generate both cytidine and uridine nucleotides, but still have protection from overall UV degradation of both materials. If we invoke the geochemical scenario postulated by Patel et al. (2015), our analysis shows that the UV environment found on the surface of the early Earth in a shallow pond or lake would allow for conversion of $\beta$-D-ribocytidine- $2^{\prime}, 3^{\prime}$-cyclic phosphate into $\beta$-D-ribouridine- $2^{\prime}, 3^{\prime}$-cyclic phosphate on appropriate timescales. The production rates of $\mathrm{C}>\mathrm{p}$ are currently not well constrained, but if production rates are nonnegligible, the UV-driven conversion of $\mathrm{C}>\mathrm{p}$ into $\mathrm{U}>\mathrm{p}$ could lead to pools of both canonical pyrimidine ribonucleotides available on the prebiotic Earth. Even if production rates are slow, the material might be protected from further loss by UV light. Potential mechanisms for UV protection could include sinking deeper into a body of water, or being otherwise shielded by other UV absorbers (Sagan, 1973). We only examined the $\mathrm{C}>\mathrm{p}$ to $\mathrm{U}>\mathrm{p}$ photoreaction in isolation in this study; in actuality on the early Earth, other potentially UV-absorbing molecules may be present. These molecules could act to shield $C>p$ and $U>p$ from UV light, or could increase the rate of degradation through various photoredox or other chemical processes. These effects are beyond the scope of this study, but they are worth noting.

UV-driven photoconversion is not the only possible way to obtain both pyrimidine ribonucleotides on the early Earth. Reaction of cytidine derivatives with nitrous acid in the dark can generate uridine derivatives (Loring and Ploeser, 1948; Shapiro and Pohl, 1968). Recent work has suggested that nitrate and nitrite (collectively called $\mathrm{NO}_{\mathrm{x}}$ ) may have been available in prebiotically relevant concentrations in shallow lake environments on the early Earth (Ranjan et al., 2019). Thus, there are seemingly multiple plausible ways in which to convert cytidine nucleotides into uridine nucleotides on the early Earth. Possibly one or more could have been at play to provide both canonical ribonucleotides for the origins of life or the development of the genetic code. Multiple ways to achieve this conversion could make it easier for uridine production to be a larger-scale process, and less confined to a specific local geochemical environment.

\section{Conclusions/Implications}

Our study determined the prebiotic plausibility of the conversion of $\beta$-D-ribocytidine- $2^{\prime}, 3^{\prime}$-cyclic phosphate into a photochemically generated intermediate that can partially convert to $\beta$-D-ribouridine- $2^{\prime}, 3^{\prime}$-cyclic phosphate, in the context of the UV light available on the surface of the early Earth. Radiation at wavelengths from 255 to $285 \mathrm{~nm}$ is most efficient at driving this reaction; such irradiation was available on the early Earth. The relative proportion of $\mathrm{C}>\mathrm{p}$ and $U>p$ after UV irradiation appears to depend modestly on the length of irradiation time, with longer times reaching similar amounts of both. However, we find that continued irradiation ( $z 1 \mathrm{~h}$ in Rayonet RPR-200, $254 \mathrm{~nm}$ ) leads to significant loss of the overall amount of $\mathrm{C}>\mathrm{p}$ and $\mathrm{U}>\mathrm{p}$ that can be recovered on heating. Proposed prebiotic chemistries for the accumulation and retention of $\mathrm{C}>\mathrm{p}$ and $\mathrm{U}>\mathrm{p}$ at, for example, $1 \mathrm{mM}$ concentrations in the surficial environment must have production rates of several to tens of micromolar per hour to be plausible. If production rates are smaller, another mechanism for UV protection must be invoked to maintain these threshold concentrations for origins of life scenarios.

\section{Acknowledgments}

The authors thank R. Szabla and D. Bucher for helpful discussions and comments. They would also like to thank V.S. Lelyveld, C.P. Tam, T.S. Walton, and S.-Y. Ng for experimental and laboratory assistance.

\section{Author Disclosure Statement}

No competing financial interests exist.

\section{Funding Information}

This work was supported in part by grants from the Simons Foundation (290363 to J.W.S. and 290360 to D.D.S.) and a fellowship from the Simons Foundation (495062 to S.R.). J.W.S. is an investigator of the Howard Hughes Medical Institute. D.D.S., S.R., and Z.R.T. would like to acknowledge funding from the Harvard Origins of Life Initiative. C.J.M. is currently at Stanford University.

\section{References}

Becker, S., Schneider, C., Okamura, H., Crisp, A., Amatov, T., Dejmek, M., and Carell, T. (2018) Wet-dry cycles enable the parallel origin of canonical and non-canonical nucleosides by continuous synthesis. Nat Commun 9:163.

Becker, S., Feldmann, J., Wiedemann, S., Okamura, H., Schneider, C., Iwan, K., Crisp, A., Rossa, M., Amatov, T., and Carell, T. (2019) Unified prebiotically plausible synthesis of pyrimidine and purine RNA ribonucleotides. Science 366:76-82.

Beckstead, A.A., Zhang, Y., de Vries, M.S., and Kohler, B. (2016) Life in the light: nucleic acid photoproperties as a legacy of chemical evolution. Phys Chem Chem Phys 18: 24228-24238. 
Cockell, C. (2000) The ultraviolet history of the terrestrial planets: implications for biological evolution. Planet Space Sci 48:203-214.

DeBoer, G., Klinghoffer, O., and Johns, H.E. (1970) Reversal mechanisms for the photohydrates of cytosine and its derivatives. Biochim Biophys Acta 213:253-268.

Ducolomb, R., Cadet, J., Taieb, C., and Teoule, R. (1976) Isomerisation and conformation studies of (+)- and (-)6-hydroxy5,6-dihydrouridine. Biochim Biophys Acta 432:18-27.

Johns, H.E., LeBlanc, J.C., and Freeman, K.B. (1965) Reversal and deamination rates of the main ultraviolet photoproduct of cytidylic acid. J Mol Biol 13:849-861.

Kim, H.-J. and Benner, S.A. (2017) Prebiotic stereoselective synthesis of purine and non canonical pyrimidine nucleotide from nucleobases and phosphorylated carbohydrates. Proc Nat Acad Sci U S A 114:11315-11320.

Lathe, R. (2006) Early tides: response to Varga et al. Icarus 180:277-280.

Lazcano, A., Guerrero, R., Margulis, L., and Oro, J. (1988) The evolutionary transition from RNA to DNA in early cells. J Mol Evol 17:283-290.

Liu, F.T. and Yang, N.C. (1978) Photochemistry of cytosine derivatives. 2. Photohydration of cytosine derivatives. Proton magnetic resonance study on the chemical structure and property of photohydrates. Biochemistry 17:4877-4885.

Loring, H.S. and Ploeser, J.M. (1948) The deamination of cytidine in acid solution and the preparation of uridine and cytidine by acid hydrolysis of yeast nucleic acid. J Biol Chem 178:439-449.

Miller, N. and Cerutti, P. (1968) Structure of the photohydration products of cytidine and uridine. Proc Natl Acad Sci 59:34-38.

Patel, B.H., Percivalle, C., Ritson, D.J., Duy, C.D., and Sutherland, J.D. (2015) Common origins of RNA, protein and lipid precursors in a cyanosulfidic protometabolism. Nat Chem 7:301-307.

Powner, M.W., Gerland, B., and Sutherland, J.D. (2009) Synthesis of activated pyrimidine ribonucleotides in prebiotically plausible conditions. Nature 459:239-242.

Ranjan, S. and Sasselov, D.D. (2016) Influence of the UV environment on the synthesis of prebiotic molecules. Astrobiology 16:68-88.

Ranjan, S. and Sasselov, D.D. (2017) Constraints on the early terrestrial surface UV environment relevant to prebiotic chemistry. Astrobiology 17:169-204.

Ranjan, S., Todd, Z.R., Rimmer, P.B., Sasselov, D., and Babbin, A.R. (2019) Nitrogen oxide concentrations in natural waters on early Earth. Geochem Geophy Geosy 20:2021-2039.

Rios, A.C. and Tor, Y. (2013) On the origin of the canonical nucleobases: an assessment of selection pressures across chemical and early biological evolution. Isr J Chem 53:469-483.

Rugheimer, S., Segura, A., Kaltenegger, L., and Sasselov, D.D. (2015) UV surface environment of earth-like planets orbiting FGKM stars through geological evolution. Astrophys $J$ 806:137.
Sagan, C. (1973) Ultraviolet selection pressure on the earliest organisms. J Theor Biol 39:195-200.

Schuster, H. (1964) Ribonucleic Acid Photochemistry. Z Naturf 19:815.

Shapiro, R. and Pohl, S.H. (1968) The reaction of ribonucleosides with nitrous acid. Side products and kinetics. Biochemistry 7:448-455.

Sinsheimer, R.L. (1957) The photochemistry of cytidylic acid. Radiation Res 6:121-125.

Sinsheimer, R.L. and Hastings, R. (1949) A reversible photochemical alteration of uracil and uridine. Science 110:525-526.

Stairs, S., Nikmal, A., Bucar, D.-K., Zheng, S.-L., Szostak, J.W., and Powner, M.W. (2017) Divergent prebiotic synthesis of pyrimidine and 8-oxo-purine ribonucleotides. Nat Commun $8: 15270$.

Todd, Z.R., Fahrenbach, A.C., Magnani, C.J., Ranjan, S., Bjorkbom, A., Szostak, J.W., and Sasselov, D.D. (2018) Solvatedelectron production using cyanocuprates is compatible with the UV-environment on a Hadean-Archaean Earth. Chem Commun 54:1121-1124.

Wang, S.Y. (1959) Ultra-violet Irradiation of 1,3dimethylthymine. Nature 184:BA59-BA61.

Wechter, W.J. and Smith, K.C. (1968) Nucleic acids. IX. The structure and chemistry of uridine photohydrate. Biochemistry 7:4064-4069.

Wierzchowski, K.L. and Shugar, D. (1961) Photochemistry of cytosine nucleosides and nucleotides. II. Acta Biochim Pol 8: 219-234.

Xu, J., Tsanakopoulou, M., Magnani, C.J., Szabla, R., Sponer, J.E., Sponer, J., Gora, R.W., and Sutherland, J.D. (2017) A prebiotically plausible synthesis of pyrimidine $\beta$-ribonucleosides and their phosphate derivatives involving photoanomerization. Nat Chem 9:303-309.

Address correspondence to:

Zoe R. Todd

Department of Astronomy

Harvard-Smithsonian Center for Astrophysics 60 Garden Street Mail-Stop 10

Cambridge, MA 02138

E-mail: zoe.todd@cfa.harvard.edu

Submitted 19 October 2019

Accepted 26 February 2020

Associate Editor: Nita Sahai

\section{Abbreviations Used}

$\mathrm{NMR}=$ nuclear magnetic resonance

$\mathrm{TMP}=$ trimethyl phosphate

$\mathrm{UV}=$ ultraviolet 


\section{Appendix}

\section{Appendix A1. Determination of Extinction Coefficients}

To convert the observed absorbances into concentrations of both the starting material and the intermediate, the extinction coefficients for these species need to be known. We assumed that the extinction coefficients for the starting material ( $\beta$-D-ribocytidine- $2^{\prime}, 3^{\prime}$-cyclic phosphate) were the same for cytidine monophosphate $\left(8814\right.$ and $9000 \mathrm{M}^{-1} \mathrm{~cm}^{-1}$ for 240 and $270 \mathrm{~nm}$, respectively). To determine the extinction coefficients for the intermediate, we obtained ${ }^{31} \mathrm{P}-\mathrm{NMR}$ spectra of a solution of $1 \mathrm{mM} \beta$-D-ribocytidine$2^{\prime}, 3^{\prime}$-cyclic phosphate before and after irradiation (254 nm, Rayonet reactor). The solution was monitored with ultraviolet (UV)-Vis absorption spectroscopy both before and after irradiation. An aliquot of the solution was spiked with a fixed concentration of trimethyl phosphate (TMP) as an internal standard to use for integration and concentration determination. The remaining unspiked solution was then irradiated in a Rayonet reactor (RPR-200, at $254 \mathrm{~nm}$ ) and monitored by UV-Vis absorption until there was some conversion to the intermediate. We then spiked this sample with the same amount of TMP and took
${ }^{31} \mathrm{P}-\mathrm{NMR}$ spectra (Varian Inova $400 \mathrm{MHz}$ spectrometer). The TMP peaks in the irradiated and unirradiated samples were used as an internal standard and to integrate the phosphorous signals from the sample. The unirradiated sample showed one peak at $17.09 \mathrm{ppm}$, whereas the irradiated sample showed two (17.09 and $17.35 \mathrm{ppm})$. Integrations of the signals were used to calculate the concentrations of the starting material and intermediate in the irradiated sample. We confirmed the identity of the starting material signal by sample spiking. With the concentrations of starting material and intermediate, as well as the absorption spectra, we could then solve for the extinction coefficients by solving the following set of equations for $\epsilon_{\mathrm{int}}, 240 \mathrm{~nm}$ and $\epsilon_{\mathrm{int}}, 270 \mathrm{~nm}$ :

$$
\begin{aligned}
& A_{240}=\epsilon_{c, 240 \mathrm{~nm}} c_{c} l+\epsilon_{\mathrm{int}, 240 \mathrm{~nm}} c_{\mathrm{int}} l \\
& A_{270}=\epsilon_{c, 270 \mathrm{~nm}} c_{c} l+\epsilon_{\mathrm{int}, 270 \mathrm{~nm}} c_{\mathrm{int}} l
\end{aligned}
$$

From this, we find that $\epsilon_{\text {int, } 240 \mathrm{~nm}}=15,875 \mathrm{M}^{-1} \mathrm{~cm}^{-1}$ and $\epsilon_{\mathrm{int}, 270 \mathrm{~nm}}=2528 \mathrm{M}^{-1} \mathrm{~cm}^{-1}$.
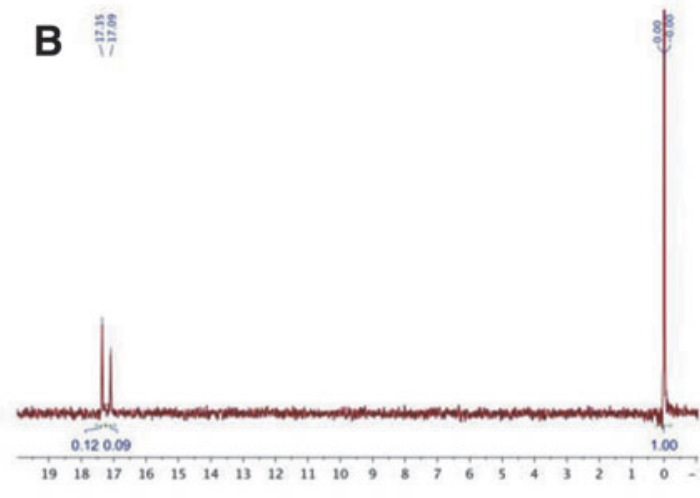

ppm

FIG. A1. (A) ${ }^{31} \mathrm{P}-\mathrm{NMR}$ spectrum of initial $2 \mathrm{mM} \beta$-D-ribocytidine- $2^{\prime}, 3^{\prime}$-cyclic phosphate, with TMP added as an internal standard. The starting material has a phosphorous signal at $17.09 \mathrm{ppm}$. (B) ${ }^{31} \mathrm{P}-\mathrm{NMR}$ spectrum of $2 \mathrm{mM} \beta$-D-ribocytidine$2^{\prime}, 3^{\prime}$-cyclic phosphate irradiated for $90 \mathrm{~min}$ in the Rayonet reactor at $254 \mathrm{~nm}$. TMP was spiked in after irradiation as an internal standard. Two signals (17.09 and $17.35 \mathrm{ppm})$ are observed; the $17.09 \mathrm{ppm}$ signal was confirmed to be the initial material by sample spiking. Integration of the two signals allowed for calculation of concentration and then extinction coefficients for the intermediate. NMR, nuclear magnetic resonance; TMP, trimethyl phosphate. 


\section{Appendix A2. Irradiation of $U>p$}

To test the properties of the uridine form of the photochemically generated intermediate, we irradiated a solution of $50 \mu \mathrm{M} \mathrm{U}>\mathrm{p}(254 \mathrm{~nm}$, Rayonet reactor) over a span of $7 \mathrm{~min}$. The UV absorption spectrum was recorded at various time intervals during the irradiation (Fig. A2). The initial $U>p$ has an absorption maximum at $260 \mathrm{~nm}$, but this feature quickly disappears with irradiation. By $7 \mathrm{~min}$, almost no $U>p$ remained. The photogenerated intermediate does not absorb significantly in the $230-300 \mathrm{~nm}$ range, which indicates that we can effectively ignore the contribution of this species to the absorption of solutions of irradiated and heated $\mathrm{C}>\mathrm{p}$. The only three species that should be detectable by UV spectroscopy are C>p, U>p, and the cytidine intermediate (absorption maxima at 270, 260 , and $240 \mathrm{~nm}$, respectively).

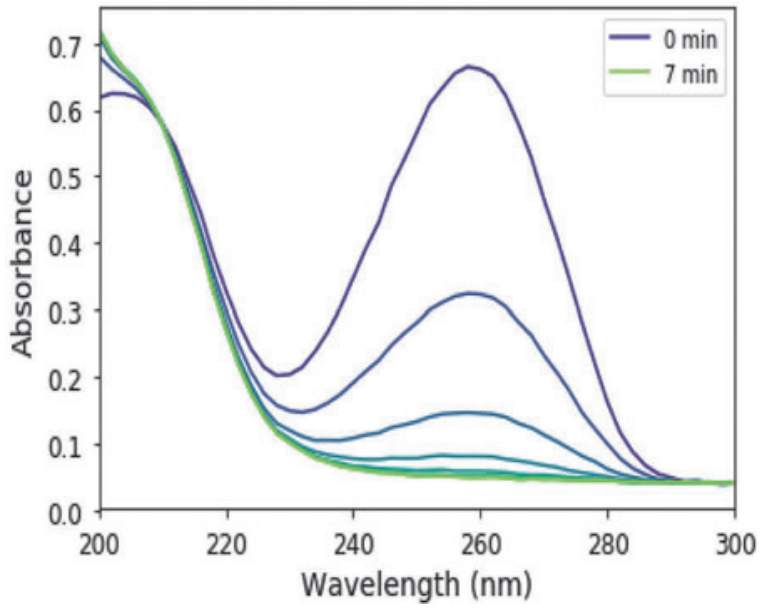

FIG. A2. UV spectra of $U>p$ during irradiation (7 min total) in the Rayonet RPR-200 $(254 \mathrm{~nm})$. The uridine photogenerated intermediate does not absorb significantly in the 230-300 nm region. UV, ultraviolet. 